Review Article

\title{
A Brief Study of Binaural Beat: A Means of Brain-Computer Interfacing
}

\author{
A. F. M. Zainul Abadin, ${ }^{1}$ Ahmed Imtiaz $\mathbb{D}^{2},{ }^{2}$ Md. Manik Ahmed, ${ }^{3}$ and Mithun Dutta ${ }^{2}$ \\ ${ }^{1}$ Department of Information and Communication Engineering, Pabna University of Science and Technology, Pabna, Bangladesh \\ ${ }^{2}$ Department of Computer Science and Engineering, Rangamati Science and Technology University, Rangamati, Bangladesh \\ ${ }^{3}$ Office of the Examination Controller, Rabindra University Bangladesh, Shahjadpur, Sirajganj, Bangladesh
}

Correspondence should be addressed to Ahmed Imtiaz; ahmedimtiaz@rmstu.edu.bd

Received 3 June 2021; Revised 29 November 2021; Accepted 30 November 2021; Published 23 December 2021

Academic Editor: Christos Troussas

Copyright $(2021$ A. F. M. Zainul Abadin et al. This is an open access article distributed under the Creative Commons Attribution License, which permits unrestricted use, distribution, and reproduction in any medium, provided the original work is properly cited.

\begin{abstract}
The human brain tends to follow a rhythm. Sound has a significant impact on our physical and mental health. This sound technology uses binaural beat by generating two tones of marginally different frequencies in each individual ear to facilitate the improved focus of attention, emotion, calming, and sensory organization. Binaural beat helps in memory boosting, relaxation, and work performance. Again because of hearing a binaural beat sound, brainwave stimuli can be diagnosed to pick up a person's sensitive information. Using this technology in brain-computer interfacing, it is possible to establish a communication between the brain and the computer. Thus, it enables us to go beyond our potential. The aim of this study is to assess the impact and explore the potential contribution of binaural beat to enhancement of human brain performance.
\end{abstract}

\section{Introduction}

Brain-computer interface (BCI) enables a nonmuscular communication between a person and an external device by measuring the brain's action. BCI is mostly used in medical science to improve the quality of life of individuals with severe neuromuscular illnesses. It is now gaining popularity in gaming, marketing, and entertainment industries. It is now used as a tool for a variety of applications such as a mind-controlled keyboard and hands-free video games or as a tool in rehabilitation. BCI-enabled games are now being developed by capturing the user current cognition state such as fulfillment, boredom, habit, and confusion [1]. One of the promising capacities of high-immersion BCI, such as a VR headset, is utilization for mood or pain management [2]. There are few studies that jointly use virtual reality and the measurement of biological variables.

Brainwave patterns have been discovered to distinguish neurocognitive states and to provide a rich feature space for researching neurological processes in both impaired and healthy users. Binaural beat has been proposed as a useful technique to change the brainwave in a noninvasive way [3]. A good amount of work has been done to find the advantages of binaural sound for brainwave control and distraction [2].

Normally, we hear sound with a combination of two ears. Monaural beat is created when two close frequency tones merge together before reaching the ears rather than merging within our brain like binaural beat [4]. The size of the species' cranium is an important factor for detecting the frequency of binaural beat. Binaural beats can be noticed in humans when carrier waves are lower than $1000 \mathrm{~Hz}$ [5]. Because the wavelength is longer than the human skull's diameter, signals below $1000 \mathrm{~Hz}$ are diffracted. Each ear gets a slightly different sound signal [6] and intensity due to its opposite position on the human skull. So, the head is not an obstacle to sounds of low pitch [7].

We anticipate that given the advancement of technology, the trend of deploying EEG-based BCI devices for nonmedical applications, such as gaming, entertainment, and marketing, will continue. Given that this technology gives information on our cognitive activity, it may be used to make 
assumptions about our goals, conscious and unconscious interests, and emotional responses.

The aim of this work is to investigate how the brain reacts to binaural beat. We summarized the effects of binaural beat simulation at different frequencies on psychophysiological measurements [8]. Following the study, we listed out how cognitive activity, in particular, attention, mood, emotion, and subconsciousness, behaves with the binaural beat in different situations such as relaxation, sleep mood, memory boosting, and task performance. Over many years, sound technology has been proposed as a healing technique to human mind. With the growth of brain-computer interface technology, it shows great potential for us. A BCI is a partnership in which the brain accepts and communicates with a mechanical device as a natural element of its representation of the body [9]. With the collection and surveyed data, the contributions of this paper are highlighted as follows:

(1) The main aspect of binaural beat and its effect on the human brain

(2) Discussion of various results that researchers have so far found

(3) A tool to collaborate with brain-computer interfacing technique to control brainwaves

\section{Literature Review}

Binaural tones and their impact on individuals have been the subject of numerous research. Binaural beat is said to have the power to alter consciousness in ways that are both paranormal and spiritual. Dove [10] initially described binaural beat events, which Thompson [11] redefined as the convergence of brain activity from the auditory nerves in binaurally sensitive networks.

Robert Monroe from the Monroe Institute was one of the first to use binaural beats for meditation on a large scale and in a business capacity [12]. However, this hypothesis has increasingly been evidenced due to scores of studies which have been released over the last couple of decades indicating high levels of consciousness in situations of low to no brain activity. In fact, high levels of consciousness have been thoroughly verified and observed in individuals who have experienced near death phenomenon, coma [13], and deep meditation [14]. Human magnetoencephalographic (MEG) research has detected auditory steady-state responses to binaural beats from various sources in the parietal, frontal, and temporal parts of the cerebral cortex including the auditory cortices [15].

BCI devices such as a neural headset and VR headset are frequently used with the binaural beat in brain relaxation therapy. A group of developers introduced a binaural beat generator android application PainDroid [16] that helps patients and therapists in objective evaluation of pain. Again, a research [17] suggests listening to a binaural beat of $6 \mathrm{~Hz}$ for 10 minutes is helpful as a meditative state inductor stimulus.

An experiment, including the psychological test, questionnaires, interviews, and observation conducted by de Wet and Potgieter [18], found out that user performance is indeed affected by sound frequencies. The result showed that, in comparison with all other frequencies, sessions where no frequencies were applied involve higher errors and longer time durations. In another study, children suffering from attention-deficit hyperactivity disorder (ADHD) were participants to see the difference in their hearing and cognitive activities [18]. The majority of these research studies concluded that binaural tones did have the power to alter the brain's dominant wave frequencies. In short, by listening to the right binaural beat, a person can achieve a higher state of relaxation, creativity, or focus [2].

\section{Binaural Beat}

There are several ways by which the brainwaves of humans change. Some of the stimuli are color, sound, music, or human contact. Now, one of the emerging techniques is binaural beat. The binaural beat phenomenon shows that the brain hears the difference between two tones coming to each ear. Binaural beats require that both of the ears hear something different. Figure 1 [19] shows a case where a left ear hears a tone of $215 \mathrm{~Hz}$ and a right ear hears $225 \mathrm{~Hz}$, where an interference pattern of $10 \mathrm{~Hz}$ binaural beat will appear. It is possible that the binaural beat produces a phase alternation and entertainment of brain oscillations, resulting in a higher neurological gain and better response to incoming inputs [20]. Solcà et al. suggested that binaural beat enhances interhemispheric coherence upon auditory listening [21]. This sort of rhythmic stimulation has the ability to change brain activity and mood and aid in relaxing the listener [22].

It is found that the tones producing beats in relatively low frequency may vary themselves in the range of $1-30 \mathrm{~Hz}$ [7] to be precise: within $18 \%$ of one another-a range called the "critical bandwidth." When two tones are tuned within $18 \%$ of one another, the brain cannot tell the difference between them and hears only one tone with a pulse [4]. However, experimentally, it is found that the human brain can be affected by up to 90 binaural beats.

\section{Impact of Binaural Beat on Brainwaves}

The human brain is an organic substance. Electrochemical energy [23] is produced during various neural activities in it. This electrochemical energy uses the electrical pulse to communicate between neurons. When a bunch of neurons generate electrical pulses, they oscillate at a certain rate of 10 to 100 cycles per second [24]. By monitoring this event in the electroencephalograph (EEG), researchers ensure the idea of brainwave pattern. Hans Berger [25] first published an article about wave-alike activities on the human brain. The scientific community so far has agreed in four categories of brainwaves by various experiments [26]. Figure 2 presents all types of brainwaves engaged in different neural activities. This indicates that a distinct brainwave will be dominant when a person is in a different mental state [18].

4.1. Boosting Cognition System and Memory. Binaural beat can be used for altering or changing our mood. It affects 


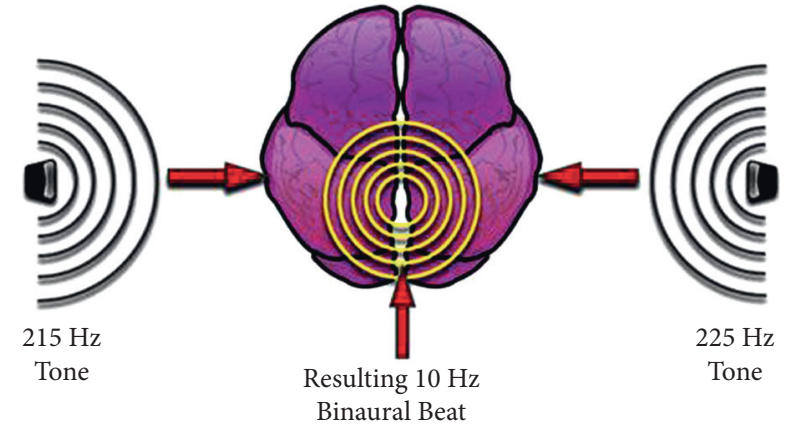

FIGURE 1: Diagram showing how binaural beat works.

breathing, heart rate, mind state [27], and feelings. Because of binaural beat, the human brain tries to oscillate at that same frequency. A neuroelectrical pulse starts sending messages at this imaginary rate [28]. Baroque music was popular in the Western world during the Renaissance era. This music consists of loud and soft long melodic combinations which are similar to alpha brainwave patterns. Listening to music, sound enters our brain stem and then the thalamus and auditory cortex, respectively [29]. It is often used in meditation, promoting relaxation and cognitive flexibility. It involves in emotion, intelligence learning, and self-healing, and its operating range should be within 30-50 BPM and 80-90 BPM [27].

During study, students try to give high focus on their subjects. That is, we shift our brainwave to a beta state. The beta state promotes more concentration producing higher frequency. When a person is unable to concentrate on something for any reason, it is recommended that he/she listens to beta binaural beats to stimulate his/her brain into the concentration mode [30]. So, the beta wave with frequencies ranging from 14 to 30 times per second implies greater effectiveness of the participant's problem-solving abilities and calculated decisions [31].

Again, a new study shows a little wakeful rest for 10 minutes after a new learning seems to be beneficial for participants [32]. It assists in accurate memory integration into the human cognitive system [33]. This type of wakeful state produces an alpha wave [34]. Besides, relaxation music promotes the brain to produce the alpha wave in the brain. Various studies have analyzed that the presence of an alpha wave promotes the secretion of $\beta$-endorphin [35-37] which aids in memory consolidation and retention [38]. So, the portions of the human brain involved in the generation of endorphins are assumed to be the same portions that are linked to memory learning [39].

Sleep disorders are becoming common in our modern world. But to have a sound cognitive function, sleep is very much essential. Researchers have proposed to use an auditory stimulus to reduce sleep disorder. One existing solution to this problem is to induce sleep using an auditory stimulus [40]. However, researchers are still learning how the sleeping process can be made faster by continuously listening to binaural beat. In fact, both sleep and relaxation improve our overall cognition, memory, and learning abilities as well as performance when we enter the theta or alpha brainwave state. When we become ready to moderate brainwaves to the alpha or theta range, it is simple to let go of old beliefs and in a real sense learn new one. This is why most of the students prefer a quiet environment to study so that they can slow their brainwaves down to theta and alpha ranges that allows to reprogram the brain. Memory boosted by endorphin injections allowed rats to run faster through mazes, according to a study [39]. Thus, more production of alpha or theta waves can be seen by practicing meditation or generating a binaural beat of $4-7.9 \mathrm{~Hz}$ or $8-13.9 \mathrm{~Hz}$. The more we listen to this frequency beat, the more the brain will be sharp.

4.2. Binaural Beats in Vigilance Task Performance. The capacity to keep one's attention focused and alert to stimuli for lengthy periods of time is known as vigilance [41]. Maintaining continuous attention, focus, and response is quite a difficult task for industrial surveillance, screening, and traffic control system. An individual's performance may decline when anybody executes an activity for a longer period of time [42].

According to research [19], low-frequency binaural beats can cause a wide range of alterations in a person's state of consciousness. Subjective relaxation can be increased by alpha frequency waves. On the contrary, attention, focus, and memory task performance are enhanced with binaural beats within the beta frequency range. A notable work has been done by Lane et al. to investigate the influence of binaural beat frequency during a vigilance task execution [43]. They found better target detection and less false alarm score by listening to beat stimulation for 30 minutes in the beta frequency range $(16 \mathrm{~Hz}$ and $24 \mathrm{~Hz})$ in comparison with the delta or theta frequency range $(1.5 \mathrm{~Hz}$ and $4 \mathrm{~Hz})$. Shoda and Burns also concluded that people who listened to beta binaural beats performed better on vigilance tasks than those who only listened to pink noise in the background [44]. In another study, $16 \mathrm{~Hz}$ beta and $7 \mathrm{~Hz}$ theta frequency ranges of binaural beats were applied by Goodin et al. in their research, and they found that the beta band would raise and maintain satisfactory alertness levels on vigilance activities [45]. According to Frederick et al., a binaural beat of $16 \mathrm{~Hz}$ to $20 \mathrm{~Hz}$ showed enhanced reaction accuracy as well as communication across brain areas [46].

Jirakittayakorn and Wongsawat conducted a study on vigilance performance by doing a word list remember task [47]. According to this study, listening to a binaural beat at $40 \mathrm{~Hz}$ for 20 minutes increased working memory performance and emotional condition. Many researchers have also agreed on that when a controlled group of patients listen to specific anxiety-decreasing music CDs, their tension decreased by a quarter amount [48]. Colzato et al. reported that listening to a $40 \mathrm{~Hz}$ gamma frequency binaural beat reduces an individual's attentional deflection [49]. Reedijk et al. also suggested that binaural auditory beats influence people's ability to concentrate their visual attention [50].

4.3. Catching Brain Stimuli in Computer by Generating Sound. In brain-computer interfaces, P300 is one of the foremost examined and utilized event-related potentials (ERPs). Due 


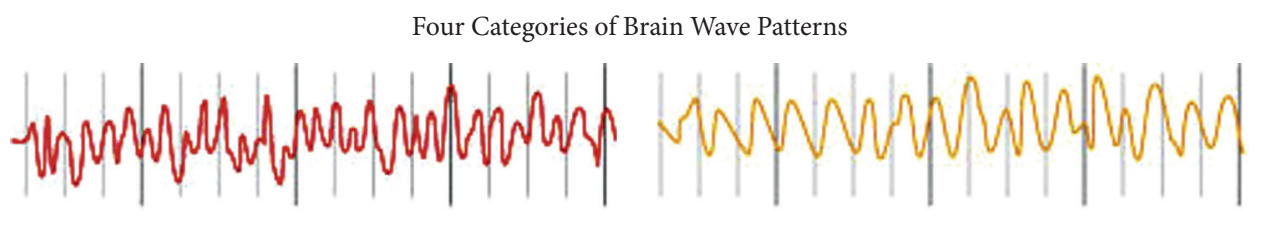

Beta $(14-30 \mathrm{~Hz})$

Concentration, arousal, alertness, cognition

Higher levels associated with anxiety, disease, feelings of separation, fight or flight

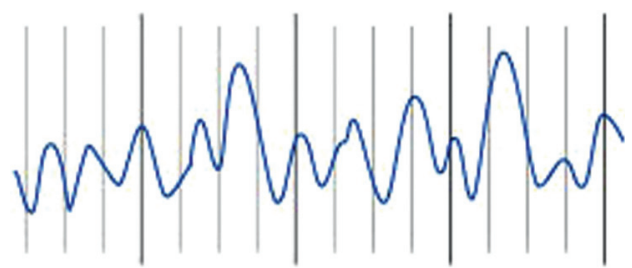

Theta $(4-7.9 \mathrm{~Hz})$

Dreaming sleep (REM sleep) Increased production of catecholamines (vital for learning and memory), increased creativity

Integrative, emotional experience, potential change in behavior, increased retention of learned material

Hypnagogic imagery, trance, deep meditation, access to unconscious mind
Alpha $(8-13.9 \mathrm{~Hz})$

Relaxation, superlearning, relaxed focus, light trance, increased serotonin production

Pre-sleep, pre-waling drowsiness, meditation, beginning of access to unconscious mind

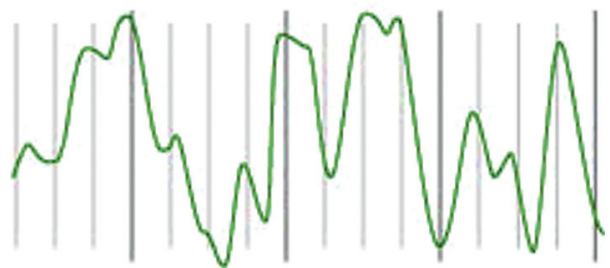

Delta $(0.1-3.9 \mathrm{~Hz})$

Dreamless sleep

Human growth hormone released

Deep, trance-like, non-physical state, loss of body awareness

Access to unconscious and "collective unconscious" mind, greatest "push" to brain when induced with Holosync ${ }^{\circledR}$

Figure 2: Categories of different brainwave patterns [19].

to any rapid potential changes in sensory, motor, or cognitive state, this signal appears on the EEG. Upon seeing or hearing something unusual, the neurons within the parietal lobe get activated. Their reaction to this unexpected stimulus results in a P300 signal [51]. This signal can usually be detected by playing an oddball paradigm in which infrequent target items are mixed with frequent nontarget items (buzz sounds, letter, picture, etc.). The P300 signal generally occurs an average of $300 \mathrm{~ms}$ after the target item in the simulation [52].

In an early study, researchers created an auditory P300based BCI using auditory stimuli like sound of "yes," "no," "pass," and "end" [53]. The usage of bell, bass, ring, thud, chord, and buzz sounds was investigated by Klobassa et al. [54]. Halder et al. also employed beep sounds to examine the effects of tone, duration, and sound source direction [55]. A study found that delivering sound from five loudspeakers in several areas brings about improved BCI execution than that obtained employing one loudspeaker [56]. Dripping sounds were studied by Huang et al., who showed that the BCI categorization precision was better when dripping sounds were utilized [57].

Listeners' brainwave activity is altered when they hear audio with binaural beats incorporated in it. It is common for mood states to be influenced by external events. Listening to a binaural beat rhythm may be a promising unused instrument for the control of cognitive processes, as well as for manipulating mood states [58]. Le Scouarnec et al. recorded binaural beats on participants' everyday activity for 30 minutes for a total of one month and found that anxiety decreased [59]. The advantages from listening to delta wave binaural beat every day for 60 days include reduced stress and anxiety and enhanced attention, concentration, and motivation, according to research conducted by Wahbeh et al. [60]. Another finding shows that binaural auditory beats alter memory, attention, and anxiety levels, as well as reducing pain perception [61]. The Profile of Mood States (POMS) questionnaire was used to measure changes in mood states by Chaieb et al. [58]. After delta wave beat stimulation, they observed a reduction in anxiety, tension, inertia, and overall mood disturbance.

Brain action corresponding to the binaural beat can be seen from the scalp [62]. Using acoustic beats and binaural beats, a study conducted by Pratt et al. found no significant difference in predicting the root of potentials if cortical activations varied with beat frequency, base frequency, and distribution [63]. As with acoustic beats, the perception of binaural beats involves similar brain activity. Binaural beat has the power to change the brainwave pattern. Listening to it for a while, a person must slightly alter his/her alertness, consciousness, and concentration. This can be a tricky way to pick up a person's brain response in front of an interrogator even though he/she will not talk. Figure 3 shows an amplitude peak called P300 in the EEG signal after 300 


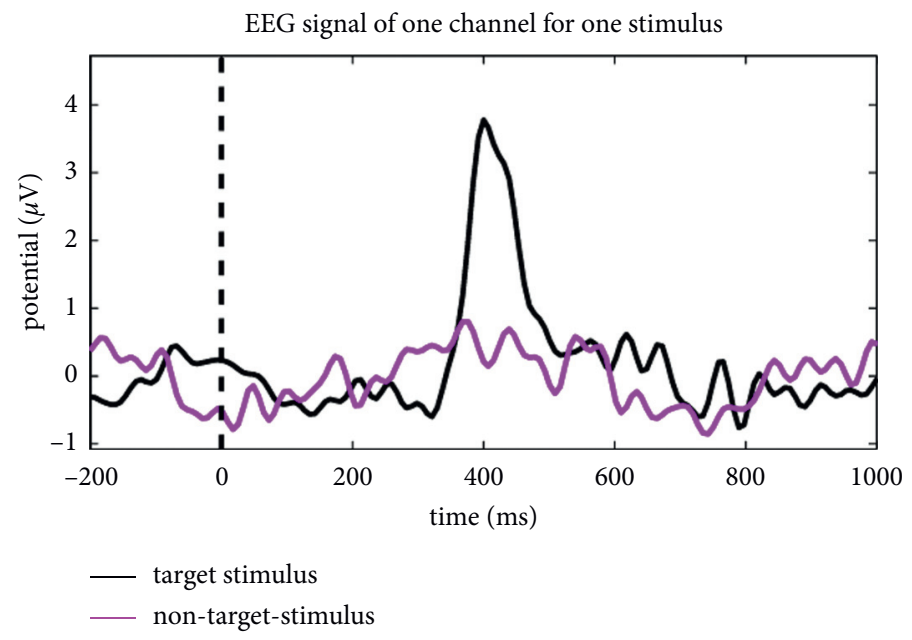

Figure 3: The P300 signal represents a matching response between the brain and a targeted picture [64].

milliseconds of the stimulus representing a matching response with the targeted information [64].

It is a very specific human brainwave pattern that occurs when anybody recognizes something meaningful or that fits his/her current task [65]. The analyst fundamentally created a program that displays images of maps, banks, and card PINs while recording each time our brain encounters P300 [64]. These data are a valuable resource to find out persons' information about their livelihood, residence, bank, etc.

For this reason, the P300 signal is considered a game changer in the field of brain-computer interfacing. The P300 signal can be divided into two subcomponents called P3a and P3b [66]. They have something to do with complicated cognitive processes like recognition and classification of external stimuli. This signal has been used to successfully identify and authenticate users. Establishing a relation between the computer and the brain has numerous applications in our daily life. In medical science, particularly for paralysis patients, we can hear thoughts on the computer. This brain-computer interfacing can revolutionize virtual reality (VR) technology. We could control any electrical equipment by our brain without touching it. This sound technology is a cheap and easy way to make a bridge between humans and computers.

\section{Discussion on Potential Uses of Binaural Beat in Brain-Computer Interfacing}

The external impact of rhythmic light and sound stimuli on the brain is known as audio-visual stimulation (AVS). Several studies look at how visual and auditory inputs interact in different brain regions. The interactions of AVS using binaural beat with flickering light are being studied by several researchers. Besle et al. [67] claimed that numerous events occur in the auditory cortex while perceiving audiovisual stimuli which may be detected by electronic measurements. According to Brauchli et al. [68], audio-visual input with differing frequencies and intensities of stimuli influences mood and autonomic arousal, but not electrocortical variables. Budzynski [69] found that a 75-year-old man's mental skills improved significantly after AVS. Cruceanu and Rotarescu [70] demonstrated that 30 minutes of AVS at a frequency of $10.2 \mathrm{~Hz}$ enhances cognitive abilities considerably. On the contrary, Kennerely [71] claimed that 5 minutes of exposure to AVS has several benefits.

At present, gadgets employing light and sound at certain frequencies are becoming popular to push the EEG towards certain frequency bands. Researchers are trying to apply the VR framework that blends the capabilities of virtual reality with other interactive media like music, binaural sound, and color in order to control temper, mood, and mental healing. Figure 4 shows a typical virtual reality (VR) headset [72] that allows the users to experience both audio and video signals at the same time. Cold colors (gray, violet, blue, etc.) are used to promote calmness, whereas hot colors (red, orange, etc.) are used to promote active brain states. Meanwhile, various binaural beats are generated to effectively impact the brainwave power. Perales et al. developed a VR environment which possesses a combination of binaural beat, color palette, background soundtrack, and scenario containing mountains, seas, and space [73]. They found a satisfactory response from the subjects in focus, alertness, and cognitive relaxation. Hence, visualization is frequently utilized as a mental training technique for the enhancement of motor abilities, inspiration, self-confidence, and strategic components in sports [74]. Besides, it helps with problem-solving and mental and pain recovery [75].

Audio-visual stimulation is believed to be reported as an viable strategy for reducing dental anxiety $[76,77]$, inducing hypnagogic mood [77], making a difference to relieve anxiety, pressure, and migraine headaches $[78,79]$, boosting up behavioral and cognitive capacities for learning disorders [80], and repairing injury from aneurysms and strokes [81]. Binaural beats and flashing color light have both been utilized to define the AVS response in the brain. The combination of binaural beats and the frequency-following flickering light stimulus has been demonstrated to affect the alpha brainwave [82]. According to Teplan and his colleagues, the cortical EEG was shown to be strongly affected 


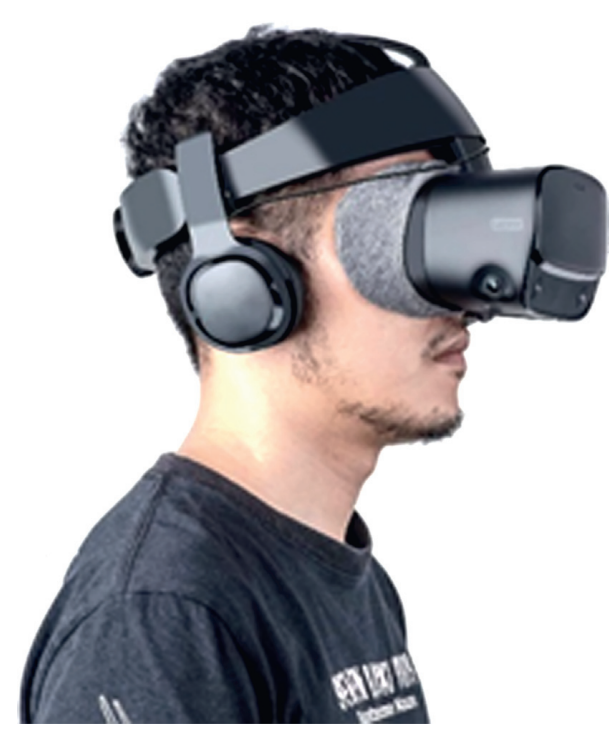

FIgURE 4: Audio-visual stimulation by applying binaural beat music in a virtual reality (VR) environment [72].

by a rectangular LED frequency-varying flashing red light stimulus with binaural beats of $2 \mathrm{~Hz}$ and $17 \mathrm{~Hz}$ [83]. Moridis et al. conducted an experiment with an $8 \mathrm{~Hz}$ binaural beat (right ear: $450 \mathrm{~Hz}$, left ear: $442 \mathrm{~Hz}$ ) paired with an $8 \mathrm{~Hz}$ flashing light in four distinct colors [82]. Thus, further research needs to be done, but a combined effect of the binaural beat with VR technology can affect brainwaves and help to drive towards a desired emotional state.

\section{Conclusion}

The goal of this study was to bridge a knowledge gap on brain functioning during binaural beats. The impact of the binaural beat is embedded deeply within our minds and produces physical changes. The interface establishes a direct link between the brain and the controlled subject. As it can alter emotion and behavior, it could be effective for arousal, self-control, attention, willingness, and performance. Recent progresses in biomedical science, computer science, and neuroscience are paving the road for braincomputer interface to become a reality, clearing the way to reestablishing and enhancing cognitive and physical capabilities. These performance-enhancing potentials might have been used in circumstances requiring sustained alertness and execution in real life. Evidence suggests that audio-visual stimulation training can help people to achieve long-term improvements in their cognitive process, concentration, alertness, and relaxation. One of the most difficult aspects of creating BCI technology has been the development of minimally invasive electrodes and surgical procedures. But the broad field of possible applications and the technological progress of auditory-based BCI devices indicate the growing interest in BCI application fields such as medical science, organizational, transportation, games, entertainment, security, and authentication. So, their pervasiveness in our everyday lives will be increased by exploring our brain's super core.

\section{Data Availability}

No data were used to support this study.

\section{Disclosure}

This research was conducted as part of authors' employment.

\section{Conflicts of Interest}

The authors declare that there are no conflicts of interest.

\section{References}

[1] A. Nijholt, "BCI for games: a state of the art" survey," in Proceedings of the 7th International Conference on Entertainment Computing (ICEC 2008), pp. 225-228, Pittsburgh, PA, USA, 2008.

[2] F. J. Perales, L. Riera, S. Ramis, and A. Guerrero, "Evaluation of A VR system for pain management using binaural acoustic stimulation," Multimedia Tools and Applications, vol. 78, no. 23, pp. 32869-32890, 2019.

[3] D. Vernon, G. Peryer, J. Louch, and M. Shaw, "Tracking EEG changes in response to alpha and beta binaural beats," International Journal of Psychophysiology, vol. 93, no. 1, pp. 134-139, 2014.

[4] Project Meditation, Binaural vs Monaural and Isochronic, LifeFlow Audio Technology, La Caillere Saint Hilaire, France, 2021, https://www.project-meditation.org/pm/binaural-vsmonaural-and-isochronic.

[5] H. W. Dove, Discovery of Binaural Beats, Binaural Blog Mindfulness Technology, Jacksonville, FL, USA, 2010, https:// www.binauralblog.com/discovery-of-binaural-beats.

[6] M. R. Rosenzweig, "Auditory localization," Scientific American, vol. 205, no. 4, pp. 132-142, 1961.

[7] G. Oster, "Auditory beats in the brain," Scientific American, vol. 229, no. 4, pp. 94-102, 1973.

[8] P. A. McConnell, B. Froeliger, E. L. Garland, J. C. Ives, and G. A. Sforzo, "Auditory driving of the autonomic nervous system: listening to theta-frequency binaural beats post-exercise increases parasympathetic activation and sympathetic withdrawal," Frontiers in Psychology, vol. 5, p. 1248, 2014.

[9] Eager_learner, Brain Computer Interface, Scribd, Inc., San Francisco, CA, USA, 2009.

[10] H. W. Dove, "Über die combination der eindrücke beider ohren und beider augen zu einem eindruck," Monatsberichte der Berliner Preussische Akademie der Wissenschaften, vol. 41, pp. 251-252, 1841.

[11] S. P. Thompson, "XXXVI. On binaural audition," The London, Edinburgh, and Dublin Philosophical Magazine and Journal of Science, vol. 4, no. 25, pp. 274-276, 1877.

[12] Amonakur, Deaf Can Hear, Cornwall, UK, 2021, http://www. jedisimon.com/articles/Deaf\%20can\%20hear.htm.

[13] J. Long, "Near-death experience. Evidence for their reality," Missouri Medicine, vol. 111, no. 5, pp. 372-380, 2014.

[14] T. Sparby, "Investigating the depths of consciousness through meditation," Mind and Matter, vol. 13, no. 2, pp. 213-240, 2015.

[15] S. Karino, M. Yumoto, K. Itoh et al., "Neuromagnetic responses to binaural beat in human cerebral cortex," Journal of Neurophysiology, vol. 96, no. 4, pp. 1927-1938, 2006.

[16] F. Spyridonis, J. Hansen, T.-M. Grønli, and G. Ghinea, "Paindroid: an android-based virtual reality application for 
pain assessment," Multimedia Tools and Applications, vol. 72, no. 1, pp. 191-206, 2014.

[17] N. Jirakittayakorn and Y. Wongsawat, "Brain responses to a $6 \mathrm{~Hz}$ binaural beat: effects on general theta rhythm and frontal midline theta activity," Frontiers in Neuroscience, vol. 11, p. 365, 2017.

[18] L. de Wet and L. Potgieter, "The impact of binaural beats on user performance and emotions using a BCI for robot control," Advances in Usability and User Experience, pp. 93-105, Springer, Cham, Germany, 2019.

[19] B. Greenfield, How You Can Use Sound and Music to Change Your Brain Waves with Laser Accuracy and Achieve Huge Focus and Performance Gains, Ben Greenfield Fitness, Washington, DC, USA, 2012, https://bengreenfieldfitness. $\mathrm{com} /$ podcast/brain-podcasts/how-you-can-use-sound-andmusic-to-change-your-brain-waves-with-laser-accuracy-andachieve-huge-focus-and-performance-gains.

[20] P. Lakatos, G. Karmos, A. D. Mehta, I. Ulbert, and C. E. Schroeder, "Entrainment of neuronal oscillations as a mechanism of attentional selection," Science, vol. 320, no. 5872, pp. 110-113, 2008.

[21] M. Solcà, A. Mottaz, and A. G. Guggisberg, "Binaural beats increase interhemispheric alpha-band coherence between auditory cortices," Hearing Research, vol. 332, pp. 233-237, 2016.

[22] S. Fessl, How Binaural Beats Affect Your Brain-and How They Don't, https://knowingneurons.com/2017/12/21/ binaural-beats, 2017.

[23] Z. Idris, "Quantum physics perspective on electromagnetic and quantum fields inside the brain," Malaysian Journal of Medical Sciences, vol. 27, no. 1, pp. 1-5, 2020.

[24] E. Dougherty, Can Brain Waves Interfere with Radio Waves? MIT School of Engineering, Cambridge, MA, USA, 2011, https://engineering.mit.edu/engage/ask-an-engineer/can-brainwaves-interfere-with-radio-waves.

[25] L. F. Haas, "Hans Berger (1873-1941), Richard Caton (1842-1926), and electroencephalography," Journal of Neurology, Neurosurgery \& Psychiatry, vol. 74, no. 1, p. 9, 2003.

[26] Z. Mohammadi, J. Frounchi, and M. Amiri, "Wavelet-based emotion recognition system using EEG signal," Neural Computing \& Applications, vol. 28, no. 8, pp. 1985-1990, 2017.

[27] J. Elder, Using Brain-Friendly Music in the Classroom, Yumpu, Diepoldsau, Switzerland, 2013, https://www.yumpu.com/en/ document/read/14989401/using-brain-friendly-music-in-theclassroom.

[28] "Society for Neuroscience," Binaural Beats Synchronize Brain Activity, Don't Affect Mood, Society for Neuroscience, Washington, DC, USA, 2020, https://www.sciencedaily.com/ releases/2020/02/200217143447.htm.

[29] S. Booth, This Is Your Brain on Binaural Beats, Healthline, San Francisco, CA, USA, 2019, https://www.healthline.com/ health-news/your-brain-on-binaural-beats.

[30] S. Cvijetic, What Are Beta Brainwaves? Improve Focus and Motivation with Beta Brainwave Entrainment, Owlcation, San Francisco, CA, USA, 2013, https://owlcation.com/ stem/What-are-Beta-Brain-Waves-Focus-and-Motivationwith-Beta-brainwave-entrainment.

[31] B. Donnelly, The Effects of Studying Music on Brain Waves Demonstrated through EEG Technology, Young Scientists Journal, 2019, https://ysjournal.com/the-effects-of-studyingmusic-on-brain-waves-demonstrated-through-eeg-technology.

[32] M. Dewar, J. Alber, C. Butler, N. Cowan, and S. Della Sala, "Brief wakeful resting boosts new memories over the long term," Psychological Science, vol. 23, no. 9, pp. 955-960, 2012.
[33] M. Craig, M. Dewar, M. A. Harris, S. Della Sala, and T. Wolbers, "Wakeful rest promotes the integration of spatial memories into accurate cognitive maps," Hippocampus, vol. 26, no. 2, pp. 185-193, 2015.

[34] R. Ramirez and Z. Vamvakousis, "Detecting emotion from EEG signals using the emotive epoc device," Brain Informatics, vol. 7670, pp. 175-184, 2012.

[35] L. Yulianty and I. Budiman, "Perbandingan pengaruh musik relaksasi dan musik yang disukai terhadap persepsi nyeri," Maranatha Journal of Medicine and Health, vol. 8, no. 2, 2009.

[36] F. Mimasa, T. Hayashi, M. Shibata, Y. Yoshitake, Y. Nishijima, and T. Moritani, "Movement of electoroencephalogram and plasm $\beta$-endorphin in the aerobic exercise," Japanese Journal of Physical Fitness and Sports Medicine, vol. 45, no. 5, pp. 519-526, 1996.

[37] Michelle Cade, Mind Like Water: Music and Wellbeing, https://www.mindlikewaterwellbeing.com/ayurvedic-soundmassage, 2019.

[38] M. Phale and D. Korgaonkar, "Pharmacology of learning and memory," The Internet Journal of Pharmacology, vol. 7, no. 1, 2008.

[39] M. A. Foster, Binaural Beats, The Bahá'í Studies Web Server, 2021, http://www.bahaistudies.net/asma/binaural.pdf.

[40] M. Lee, C.-B. Song, G.-H. Shin, and S.-W. Lee, "Possible effect of binaural beat combined with autonomous sensory meridian response for inducing sleep," Frontiers in Human Neuroscience, vol. 13, p. 425, 2019.

[41] J. S. Warm, R. Parasuraman, and G. Matthews, "Vigilance requires hard mental work and is stressful," Human Factors: The Journal of the Human Factors and Ergonomics Society, vol. 50, no. 3, pp. 433-441, 2008.

[42] E. L. Wiener, "Application of vigilance research: rare, medium, or well done?" Human Factors: The Journal of the Human Factors and Ergonomics Society, vol. 29, no. 6, pp. 725-736, 1987.

[43] J. D. Lane, S. J. Kasian, J. E. Owens, and G. R. Marsh, "Binaural auditory beats affect vigilance performance and mood," Physiology \& Behaviour, vol. 63, no. 2, pp. 249-252, 1998.

[44] E. A. Shoda and G. N. Burns, "The impact of binaural beat technology on vigilance task performance, mental workload, and psychological stress," in Proceedings of the 17th International Symposium on Aviation Psychology, pp. 459-464, Dayton, OH, USA, 2013.

[45] P. Goodin, J. Ciorciari, K. Baker, A.-M. Carrey, M. Harper, and J. Kaufman, "A high-density EEG investigation into steady state binaural beat stimulation," PLoS One, vol. 7, no. 4, Article ID e34789, 2012.

[46] J. A. Frederick, J. F. Lubar, H. W. Rasey, S. A. Brim, and J. Blackburn, "Effects of $18.5 \mathrm{~Hz}$ auditory and visual stimulation on EEG amplitude at the vertex," Journal of Neurotherapy, vol. 3, no. 3-4, pp. 23-28, 1999.

[47] N. Jirakittayakorn and Y. Wongsawat, "Brain responses to $40 \mathrm{~Hz}$ binaural beat and effects on emotion and memory," International Journal of Psychophysiology, vol. 120, pp. 96-107, 2017.

[48] C. Adams, Do Binaural Beats Change Your Mood?, https:// www.cityweekly.net/utah/rock-a-bye-bb/Content? oid=2147564, 2010.

[49] L. S. Colzato, H. Barone, R. Sellaro, and B. Hommel, "More attentional focusing through binaural beats: evidence from the global-local task," Psychological Research, vol. 81, no. 1, pp. 271-277, 2017. 
[50] S. A. Reedijk, A. Bolders, L. S. Colzato, and B. Hommel, "Eliminating the attentional blink through binaural beats: a case for tailored cognitive enhancement," Frontiers in Psychiatry, vol. 6, p. 82, 2015.

[51] "Experiment: the consciousness detector-EEG, oddball task, and P300 ," Backyard Brains, Ann Arbor, MI, USA, 2017, https://backyardbrains.com/experiments/p300.

[52] S. Sutton, M. Braren, J. Zubin, and E. R. John, "Evoked-potential correlates of stimulus uncertainty," Science, vol. 150, no. 3700, pp. 1187-1188, 1965.

[53] E. W. Sellers and E. Donchin, "A P300-based brain-computer interface: initial tests by ALS patients," Clinical Neurophysiology, vol. 117, no. 3, pp. 538-548, 2006.

[54] D. S. Klobassa, T. M. Vaughan, P. Brunner et al., "Toward a high-throughput auditory P300-based brain-computer interface," Clinical Neurophysiology, vol. 120, no. 7, pp. 1252-1261, 2009.

[55] S. Halder, M. Rea, R. Andreoni et al., "An auditory oddball brain-computer interface for binary choices," Clinical Neurophysiology, vol. 121, no. 4, pp. 516-523, 2010.

[56] M. Schreuder, B. Blankertz, and M. Tangermann, "A new auditory multi-class brain-computer interface paradigm: spatial hearing as an informative cue," PLoS One, vol. 5, no. 4, Article ID e9813, 2010.

[57] M. Huang, J. Jin, Y. Zhang, D. Hu, and X. Wang, "Usage of drip drops as stimuli in an auditory P300 BCI paradigm," Cognitive Neurodynamics, vol. 12, no. 1, pp. 85-94, 2018.

[58] L. Chaieb, E. C. Wilpert, T. P. Reber, and J. Fell, "Auditory beat stimulation and its effects on cognition and mood states," Frontiers in Psychiatry, vol. 6, p. 70, 2015.

[59] R. P. Le Scouarnec, R. M. Poirier, J. E. Owens, J. Gauthier, A. G. Taylor, and P. F. Rodeheaver, "Use of binaural beat tapes for treatment of anxiety: a pilot study of tape preference and outcomes," Alternative Therapies in Health and Medicine, vol. 7, no. 1, pp. 58-63, 2001.

[60] H. Wahbeh, C. Calabrese, and H. Zwickey, "Binaural beat technology in humans: a pilot study to assess psychologic and physiologic effects," Journal of Alternative \& Complementary Medicine, vol. 13, no. 1, pp. 25-32, 2007.

[61] M. Garcia-Argibay, M. A. Santed, and J. M. Reales, "Efficacy of binaural auditory beats in cognition, anxiety, and pain perception: a meta-analysis," Psychological Research, vol. 83, no. 2, pp. 357-372, 2019.

[62] H. Pratt, A. Starr, H. J. Michalewski, A. Dimitrijevic, N. Bleich, and N. Mittelman, "Cortical evoked potentials to an auditory illusion: binaural beats," Clinical Neurophysiology, vol. 120, no. 8, pp. 1514-1524, 2009.

[63] H. Pratt, A. Starr, H. J. Michalewski, A. Dimitrijevic, N. Bleich, and N. Mittelman, "A comparison of auditory evoked potentials to acoustic beats and to binaural beats," Hearing Research, vol. 262, no. 1-2, pp. 34-44, 2010.

[64] I. Martinovic, D. Davies, M. Frank, D. Perito, T. Ros, and D. Song, "On the feasibility of side-channel attacks with braincomputer interfaces," in Proceedings of the 21st USENIX conference on Security symposium (Security'12), p. 34, Bellevue, WA, USA, 2012.

[65] J. Leach, Brain Hacking: A New Reality?, Seckman High School Prowl Newspaper, Imperial, MO, USA, 2014, https://shsprowlnewspaper.blogspot.com/2014/11/brainhacking-new-reality. html.

[66] J. Polich, "Updating P300: an integrative theory of P3a and P3b," Clinical Neurophysiology, vol. 118, no. 10, pp. 2128-2148, 2007.
[67] J. Besle, O. Bertrand, and M.-H. Giard, "Electrophysiological (EEG, sEEG, MEG) evidence for multiple audiovisual interactions in the human auditory cortex," Hearing Research, vol. 258, no. 1-2, pp. 143-151, 2009.

[68] P. Brauchli, C. M. Michel, and H. Zeier, "Electrocortical, autonomic, and subjective responses to rhythmic audio-visual stimulation," International Journal of Psychophysiology, vol. 19, no. 1, pp. 53-66, 1995.

[69] T. Budzynski and H. Budzynski, "Brain brightening-preliminary report," in House ManuscriptMind Alive Inc., Edmonton, Canada, 2001.

[70] D. V. Cruceanu and S. V. Rotarescu, "Alpha brainwave entrainment as a cognitive performance activator," Cognition, Brain, Behavior, vol. 17, no. 3, pp. 249-261, 2013.

[71] R. Kennerely, "QEEG analysis of binaural beat audio entrainment: a pilot study," Journal of Neurotherapy, vol. 8, pp. 122-127, 2004.

[72] MYJK Stereo VR Headphone/Soundkit Custom Made for Oculus Rift S VR Headset-1 Pair (2021 New Version), Amazon, Washington, DC, USA, 2020, https://www.amazon. com/MYJK-Professional-Headphone-Headset-1-Installing/ $\mathrm{dp} / \mathrm{B} 08 \mathrm{~W} 25 \mathrm{~T} 2 \mathrm{FD}$ ?th $=1$.

[73] F. J. Perales, M. Sanchez, L. Riera, and S. Ramis, "A pilot study: VR and binaural sounds for mood management," in Proceedings of the 2018 22nd International Conference Information Visualisation (IV), pp. 442-447, Fisciano, Italy, 2018.

[74] T. Kajtna, T. Jeromen, and M. Kočevar, Sport with a Clear Head: Snapshots from Sports Psychology for Young Athletes, Self-Publishing, Ljubljana, Slovenia, 2013.

[75] V. Nagar and M. Noohu, "The effect of mental imagery on muscle strength and balance performance in recreational basketball players," Medicina Sportiva, vol. 10, no. 3, pp. 2387-2393, 2014.

[76] D. R. Morse, "Brain wave synchronizers: a review of their stress reduction effects and clinical studies assessed by questionnaire, galvanic skin resistance, pulse rate, saliva, and Electroencephalograph," Stress Medicine, vol. 9, no. 2, pp. 111-126, 1993.

[77] J. N. I. Dieter and J. A. Weinstein, "The effects of variable frequency photo-stimulation goggles on EEG and subjective conscious state," Journal of Mental Imagery, vol. 19, no. 1-2, pp. 77-89, 1995.

[78] G. D. Solomon, "Slow wave photic stimulation in the treatment of headache-a preliminary report," Headache: The Journal of Head and Face Pain, vol. 25, no. 8, pp. 444-446, 1985.

[79] D. J. Anderson, "The treatment of migraine with variable frequency photo-stimulation," Headache: The Journal of Head and Face Pain, vol. 29, no. 3, pp. 154-155, 1989.

[80] J. L. Carter and H. L. Russell, "A pilot investigation of auditory and visual entrainment of brainwave activity in learningdisabled boys," Texas Research, vol. 4, pp. 65-73, 1993.

[81] H. L. Russell, "Intellectual, auditory and photic stimulation changes in functioning in children and adults," Biofeedback, vol. 25, no. 1, pp. 16-17, 1997.

[82] C. N. Moridis, M. A. Klados, I. A. Kokkinakis et al., "The impact of audio-visual stimulation on alpha brain oscillations: an EEG study," in Proceedings of the 10th IEEE International Conference on Information Technology and Applications in Biomedicine, pp. 1-4, Corfu, Greece, 2010.

[83] M. Teplan, A. Krakovská, and S. Štolc, "Direct effects of audiovisual stimulation on EEG," Computer Methods and Programs in Biomedicine, vol. 102, no. 1, pp. 17-24, 2011. 